\title{
12. Trasplante pulmonar en pacientes con fibrosis pulmonar idiopática
}

\author{
CATALINA BRICEÑO V.*, CLAUDIA SEPÚLVEDA L.** y JOEL MELO T.***
}

\section{Lung transplant in patients with idiopathic pulmonary fibrosis}

Pulmonary transplantation represents a therapeutic option in patients with advanced pulmonary fibrosis who have a progressive course despite medical treatment, improving their survival and quality of life. It is vital that the pulmonologist knows when to refer a patient and be familiar with the requirements and contraindications. Timely referral grants the necessary time for a complete study, in addition to identifying contraindications and clinical conditions that could be modified and / or corrected before a transplant.

Key words: Pulmonary Fibrosis; Quality of Life; Transplants; Referral and Consultation.

\section{Resumen}

El trasplante pulmonar representa una opción terapéutica en pacientes con fibrosis pulmonar avanzada que presentan un curso progresivo a pesar de tratamiento médico, mejorando su sobrevida y calidad de vida. Es de vital importancia que el neumólogo conozca cuando referir a un paciente y estar familiarizado con los requisitos necesarios y contraindicaciones para enviar un paciente a un centro de trasplante. La derivación oportuna permite realizar un completo estudio, además de identificar las contraindicaciones y condiciones clínicas susceptibles de modificar y/o corregir antes de un trasplante.

Palabras clave: Fibrosis pulmonar; Calidad de Vida; Trasplante; Referencia y Evaluación.

El trasplante pulmonar es una opción terapéutica en pacientes con fibrosis pulmonar avanzada que presentan un curso progresivo a pesar de tratamiento médico, mejorando su sobrevida y calidad de vida. La derivación oportuna a un centro de trasplante otorga el tiempo necesario para un completo estudio del potencial candidato, además permite identificar contraindicaciones y condiciones clínicas susceptibles de modificar y/o corregir antes de un trasplante ${ }^{1}$. El momento de la derivación es especialmente importante en pacientes con fibrosis pulmonar idiopática (FPI), donde la sobrevida media al momento del diagnóstico es de 2 a 3 años y sólo un 20 a $30 \%$ de los pacientes sobreviven más de 5 años luego del diagnóstico.
A continuación se enumeran los criterios de derivación a una unidad de trasplante y los criterios de inclusión en una lista de espera a trasplante ${ }^{2}$.

\section{Criterios de derivación}

1. Evidencia radiológica y/o histológica definitivas de patrón de neumonía intersticial usual (NIU), independiente de la función pulmonar.

2. Pruebas de función pulmonar alteradas: capacidad vital forzada $(\mathrm{CVF})<80 \%$ del predicho o DLco $<40 \%$ del predicho.

3. Disnea o limitación funcional.

4. Necesidad de oxigenoterapia, incluso si es sólo durante ejercicio.

* Hospital Clínico Pontificia Universidad Católica de Chile. Santiago, Chile.

** Instituto Nacional del Tórax y Clínica Santa María. Santiago, Chile.

***Instituto Nacional del Tórax y Clínica Las Condes. Santiago, Chile. 


\section{Criterios de inclusión en lista de espera}

1. Caída de la $\mathrm{CVF} \geq 10 \%$ durante 6 meses de seguimiento.

2. Caída de la DLco $\geq 15 \%$ durante 6 meses seguimiento.

3. Desaturación de oxígeno $<88 \%$ o distancia recorrida $<250$ metros durante TC6M, o caída $>50 \mathrm{~m}$ en un período de 6 meses.

4. Hipertensión pulmonar en ecocardiograma o cateterismo derecho.

5. Hospitalización por progresión de enfermedad, neumotórax o exacerbación aguda.

\section{Algunas consideraciones especiales}

El neumólogo además de conocer cuando referir a un paciente, debe estar familiarizado con los requisitos y contraindicaciones necesarias para enviar un paciente a un centro de trasplante, las cuales se describen a continuación:

\section{Contraindicaciones absolutas}

1. Diagnóstico de cáncer en los últimos 5 años, con excepción de tumores de piel basocelular o escamoso (período libre de enfermedad 2 años).

2. Disfunción intratable de otros órganos vitales.

3. Enfermedad coronaria no susceptible a revascularización.

4. Inestabilidad médica aguda (sepsis, infarto agudo del miocardio, falla hepática), diátesis hemorrágica.

5. Infección activa por Mycobacterium tuberculosis.

6. Deformidad significativa de la pared torácica o espinal.

7. Obesidad clase II o III (IMC $\geq 35$ ).

8. Falta de adherencia a tratamiento.

9. Condición psiquiátrica o psicológica que inhabilite.

10. Ausencia o inadecuada red de soporte social.

11. Estado funcional severamente limitado con pobre potencial de rehabilitación.

12. Tabaquismo o adicción activa o en los últimos seis meses.

\section{Contraindicaciones relativas *}

1. Edad $>65$ años (alrededor de esta edad el paciente debe estar en buen estado general y no tener comorbilidades significativas).

2. Obesidad clase I (IMC 30-34,9).

3. Desnutrición severa (IMC < 17).

4. Cirugía pulmonar extensa con resección pulmonar.

5. Osteoporosis severa y sintomática.

6. Estar con ventilación mecánica o con oxigenación extracorpórea-ECMO (deberá ser evaluado caso a caso por equipo multidisciplinario del centro).

7. Colonización por gérmenes resistentes (Mycobacterium no TBC).

8. Infección por hepatitis $\mathrm{B}$ y $\mathrm{C}$, sin signos de cirrosis ni hipertensión portal.

9. Infección VIH (debe ser considerado algo excepcional será evaluado caso a caso por equipo de trasplante).

10. Infección por Burkholderia cenocepacia, Burkholderia gladioli (gran parte de los programas lo considera una contraindicación absoluta).

Si bien estos puntos son considerados contraindicaciones relativas por la Sociedad Internacional de Trasplante de Corazón y Pulmón la decisión final de ingreso a lista será del equipo multidisciplinario del centro de referencia de trasplante

\section{Lugar de residencia}

Podrán ser candidatos a trasplante pulmonar todos los pacientes que en el estudio pre-trasplante sean considerados idóneos. Sin embargo, al momento de ingresar en la lista de espera nacional, deben residir en Santiago o dentro de un radio de no mayor a 200 kilómetros, con la posibilidad de llegar al centro de trasplante en menos de $2 \mathrm{~h}$ si existe un donante. Luego del trasplante el paciente debe permanecer en Santiago o en un radio no mayor a 200 kilómetros por un período no inferior a 6 meses.

\section{Bibliografía}

1.- WEILL D, BENDEN C, CORRIS PA, DARK JH, DAVIS RD, KESHAVJEE S, et al. A consensus document for the selection of lung transplant candidates: 2014 An update from the Pulmonary Transplantation Council of the international society for Heart and lung Transplantation. J Heart lung Transplant 2015; 34: 1-15.

2.- YUSEN RD, EDWARDS LB, DIPCHAND AI, GOLDFARB SB, KUCHERYAVAYA AY, LEVVEY B, et al. The registry of the international society for Heart and lung Transplantation: Thirty-third adult lung and Heart-lung Transplant report-2016; Primary diagnostic indications for Transplant. J Heart lung Transplant 2016; 35: $1170-84$.

Correspondencia a:

Dra. Catalina Briceño V.

Email: catalinabriceno@gmail.com 